\title{
Therapeutic anti-tumor effect of exogenous apoptin driven by human survivin gene promoter in a lentiviral construct
}

\author{
Feng Ye ${ }^{1}$, Bo Zhong 2 , Guorong Dan¹, Fan Jiang', Yan Sai', Jiqing Zhao', Huiqin Sun ${ }^{3}$, Zhongmin Zou ${ }^{1}$
}

\author{
${ }^{1}$ Institute of Toxicology, School of Preventive Medicine, Third Military Medical \\ University, Chongqing, China \\ 2Department of Oncology, Southwest Hospital, Chongqing, China \\ 3 Institute of Combined Injury, State Key Laboratory of Trauma, Burn and Combined \\ Injury, Third Military Medical University, Chongqing, China
}

\author{
Submitted: 17 January 2012 \\ Accepted: 11 May 2012
}

Arch Med Sci 2013; 9, 3: 561-568

DOI: 10.5114 /aoms.2013.35423

Copyright $\odot 2013$ Termedia \& Banach

\begin{abstract}
Introduction: The aim of this study was to construct a lentivirus vector with survivin promoter (pSur)-driven apoptin and test its efficiency in suppressing the growth of tumor cells.

Material and methods: Expression cassettes with different fragments of survivin gene promoter (pSur, 161 bp, 272 bp, 990 bp) driving 6XHis-tagged apoptin were constructed to generate recombinant lentivirus, of which the inhibitory effect on tumor cells was compared. The activity of different pSur in 293FT, and 272 bp pSur in primary bone marrow mesenchymal stem cells (BMSCs), SW480, Hela and MCF-7 was examined by Western blot. Their ability to induce apoptosis in SW480 cells was determined by annexin-V staining. The inhibitory effect of letivirus containing different pSur-driven apoptin on nude mice-xenografted SW480 cells was assessed by tumor size and pathological observation.

Results: The 272 bp and 990 bp pSur displayed comparable effects in terms of promoter activity, cell apoptosis/necrosis and G1 phase arrest in vitro, and growth of xenograft tumor in vivo. When lentivirus containing 272 bp pSur was tested, it drove high apoptin expression in tumor cells (SW480, Hela and MCF-7) and weak expression in primary bone marrow mesenchymal stem cells. Xenograft to nude mice using infected Sw480 cells showed that lentiviruses possessing $272 \mathrm{bp}$ and $990 \mathrm{bp}$ pSur were able to significantly induce tumor cell death, focal necrosis, and tumor growth lag.

Conclusions: The data indicated that pSur-apoptin expression cassette in lentivirus vector ensures specific suppression of tumor cells, and may be applicable to monitor malignant transformation of transplanted cells.
\end{abstract}

Key words: gene therapy, lentivirus, survivin promoter, apoptin, tumor suppression.

\section{Introduction}

The applicability of gene therapy for cancer is dependent on its efficiency in tumor cell elimination and specificity to target tumor cells. The specificity will ensure the killing of malignant cells while not hurting normal cells. Apoptin, a chicken anemia virus-derived protein, can specifically induce apoptosis of various tumor cells by translocating to the nucleus of tumor cells, and targeting DEDAF, Nur77, Nmi, Hippi and APC1 [1-3]. Cellular associations through apoptin and its binding partners impulse the

\author{
Corresponding authors: \\ Zhongmin Zou \\ Institute of Toxicology \\ School of Preventive Medicine \\ Third Military \\ Medical University \\ Chongqing 400038, China \\ Phone: 86-23-68752285 \\ Fax: 86-23-68752285 \\ E-mail: zmzou@tmmu.edu.cn, \\ zouzhmin@yahoo.com \\ Huiqin Sun \\ Institute of Combined Injury \\ State Key Laboratory of Trauma, \\ Burn and Combined Injury \\ Third Military \\ Medical University \\ Chongqing 400038, China \\ Phone: 86-23-68771724 \\ Fax: 86-23-68771724 \\ E-mail: \\ huiqinsun02@yahoo.com.cn
}


death signal to different signal transduction pathways and finally lead to the death of the host cancer cells $[4,5]$. Apoptin can also induce tumor apoptosis in cases where (chemo) therapy might fail, due to its p53-independent action, and insensitivity to BCR-ABL and Bcl-2 [4, 6]. Apoptin has been constructed into several types of vectors for cancer gene therapy, and exhibited a significant tumorsuppressing effect $[7,8]$.

In gene therapy, promoter controls the expression of an exogenous gene with suitable time and level in a specific population of cells. Survivin, a member of the inhibitor of apoptosis protein (IAP) family, counteracts cell death and regulates mitotic progression, and is activated in most types, if not all, of tumor cells but not in normal cells $[9,10]$. So, survivin promoter (pSur) has been tested as a transcriptional targeting strategy for tumor treatment [11-14], and showed a potent anti-tumor effect by driving the expression of tumor-specific short hairpin RNA (shRNA) [12], suicide genes [13], and sodium/iodide symporter [14]. We presume that a pSurdriven apoptin expression cassette will preferentially be activated in tumor cells and malignantly transformed cells, and induce apoptosis of the cells, leaving normal cells unaffected.

Lentivirus is an important and efficient delivery vector in cancer gene therapy [15]. In the present study, three pSur fragments with different lengths were separately used to drive apoptin expression, and pSur transcriptional activity and apoptosis-inducing ability were examined in lentivirus-infected cells. This strategy is practically useful in counteracting malignant cells by self-judged activation of pSur, the expression of apoptin and the elimination of unwanted cells via cell apoptosis. Our self-surveillance and self-elimination strategy of gene therapy may be applied to monitor the malignant transformation of transplanted stem cells, such as in the management of leukemia and wound healing [16].

\section{Material and methods}

\section{Cell lines}

The lentivirus packaging cell line 293FT, human colon cancer cell line SW480, human breast cancer cell line MCF-7, and human epithelial cervical cancer line Hela were originally purchased from the American Type Culture Collection (ATCC, Rockville, MD) and kept in this lab. Cells were maintained at $37^{\circ} \mathrm{C}$ in a humidified atmosphere containing $5 \% \mathrm{CO}_{2}$ in Dulbecco's Modified Eagle Medium (high glucose) medium supplemented with 10\% FBS and $100 \mathrm{IU} /$ $\mathrm{ml}$ penicillin and $100 \mathrm{ng} / \mathrm{ml}$ streptomycin. Human bone marrow samples were collected from normal volunteers $(n=3)$ with paper consent and approved by the ethical committee of the institution (Southwest Hospital, Chongqing, China). Primary bone marrow mesenchymal stem cells (BMSC) were cultured as reported before [17].

\section{Cloning of human survivin gene promoter with different lengths}

Human pSur was amplified by PCR on genomic DNA from BMSC. The lengths of the amplified fragments of human pSur were 161 bp, 272 bp and $990 \mathrm{bp}$. The sequences of the oligonucleotide primers were as follows: forward primer, pSur161-F 5'-TGCCTCGAGTACAACTCCCGGCCACAC-3', pSur272-F 5'-TGCCTCGAGCACGCGTTCTTTGAAAGC-3', pSur990F 5'-TGCCTCGAGCCTGGCCATAGAACCAGAGAAGTG3', and the shared common reverse primer, pSurR 5'-GCCGCGGCCGCCACCTCTGCCAACG-3'. Restriction enzyme (RE) cutting sites for Xho I and Not I were introduced (underlined in the respective sequences). The PCR amplified pSur was TA cloned into PMD-T18 vector (Takara, Dalian, China) for sequencing and named pMD-T161pSur, pMDT272pSur, and pMD-T990pSur, respectively.

\section{Construction of recombinant lentiviral constructs with pSur-driven apoptin expression}

Apoptin cDNA was amplified from T-apoptin plasmid (a kind gift from Dr. Bin Yang, Institute of Agriculture and Forestry Sciences, Beijing, China) by PCR with the primer sequences as: Apo-S 5'-TGGCGGCCGCGGCATGAACGCTCTCCAAGAAG-3', Apo-AS 5'-CAGATCTAGAATTAATGGTGATGGTGATGATGCAGTCTTATACACCTTCT-3'. Underlined nucleotides were cutting sites for Not I and Xba I, and $6 \times \mathrm{His}$ tag. The PCR product Apo- $6 \times$ His fragment was subcloned downstream of the pSur in PMD-TpSur between Not I and Xba I sites. The plasmids were verified and named as PMD-TpSur-ApoHis.

FG12 (generously provided by Dr. Baltimore, California Institute of Technology, CA, USA), a second generation self-inactivation (SIN) lentivirus vector, was used as the lentiviral backbone. The ubiquitin$C$ promoter ( $\mathrm{pUbiC}$ ) in this vector independently drives the reporter green fluorescent protein (GFP) expression, which indicates the lentivirus-infected cells. A polyA sequence downstream of the exogenous gene was added at the multiple cloning sites of FG12. Two complementary DNA oligos were synthesized, annealed, and ligated to Xba I-linearized FG12 vector. The sequences of the DNA oligos were as follows: PolyA-S 5'-CTAGAGGATCCAATAAAGGATCTTTTATTTTCATTGGATCTGTGTGTTGGTTTTTTGTATA-3' and PolyA-AS 5'-CTAGTATACAAAAAACCAACACACAGATCCAATGAAAATAAAAGATCCTTTATTGGATCC T-3'. Xba I adhesive ends at both ends of the annealed double strand DNA and BamH I (underlined in the respective sequences) were introduced.

Finally, the pSur-driven apoptin-6xHis expressing cassette in pMD-TpSur-apoHis was subcloned 
into modified FG12 between Xho I and Xba I sites. The plasmids named as FG-161/272/990pSurapoHis were confirmed by RE digestion and sequencing.

\section{Comparing the activity of different pSur by transient transfection}

To compare the activity of different pSur in FGpSur-ApoHis, the recombinant lentivirus vectors were transiently transfected into 293FT cells while FG12 without insert was used as a negative control. Briefly, $1 \times 10^{6} 293 \mathrm{FT}$ cells were plated in 6-well tissue culture plates, and $24 \mathrm{~h}$ later, the cells were transfected with $0.2 \mu \mathrm{g}$ of indicated plasmids plus $0.2 \mu \mathrm{g}$ of pMIR-Report $\beta$-gal plasmid (Ambion) as an internal control using Lipofectamine 2000 (Invitrogen) according to the manufacturer's protocol. Cell lysates were harvested $48 \mathrm{~h}$ later. The expression of $\beta$-gal was detected using luciferase detection kit (Beyotime, China), and apoptin-6xHis was detected by Western blot (see below) to assess the activity of different pSur.

\section{Lentivirus production and infection}

All lentiviruses were produced by cotransfecting the lentiviral vector FG-pSur-ApoHis, the HIV-1 packaging vector D8. 9, and the VSVG envelope glycoprotein into 293FT cells as reported [18]. The viruses were collected from the culture supernatants on days 2 and 3, followed by ultracentrifugation concentration. The concentrated virus stocks were titrated on 293 FT cells based on GFP expression. The lentivirus were named as Lenti-161/272/ 990pSur-ApoHis and Lenti-FG12 respectively.

For lentivirus infection, BMSC, Hela, MCF-7, and SW480 cells were plated $24 \mathrm{~h}$ prior to infection and reached about $50 \%$ degrees of confluence before the infection. The cells were then incubated with fresh media containing required multiplicity of infection (MOI) of virus for $24 \mathrm{~h}$. All lentivirus infections were performed in the presence of $8 \mu \mathrm{g} / \mathrm{ml}$ polybrene (Sigma, Poole, UK). GFP expression was visualized by fluorescence microscopy at 5 day after the infection.

\section{Western blot against apoptin-His protein}

After liposomal transfection of lentivirus vectors or lentivirus infection, cells were collected at the indicated time for apoptin detection by Western blot with primary antibody against $6 \times \mathrm{His}$ tag (mouse anti-HIS, $1: 1000$, Qiangen, China) and secondary antibody (Goat anti-mouse HRP, $1: 1000$, Boster, China). Bands were visualized with $\mathrm{ECL}$ Western Blotting Substrate (Beyotime, China). Extracts of the lentiviral vector (FG12) or lentivirus (Lenti-FG) treated cells were used as a negative control and $\beta$-actin was used as an internal control. All measurements were performed in triplicate.

\section{Apoptosis detection}

Apoptosis of SW480 was detected at 5 day and 30 day after the lentivirus infection. Cells were washed twice with PBS, followed by adding Annexin V-PE, 7-AAD as instructed. Apoptosis and necrosis were observed under fluorescence microscopy. Annexin V-PE could bind to the membrane of apoptotic and necrotic cells, showing red color. 7-AAD could specifically bind to necrotic cell nuclei, showing bright orange color. At least three representative pictures for each treatment were chosen, and the numbers of apoptosis and necrosis per 1000 cells were determined by visual examination.

\section{Cell cycle analysis with flow cytometry}

After the lentivirus infection $(\mathrm{MOI}=20)$, SW480 cells in a 6-plate panel were harvested ( $80 \%$ confluence) at 5 day and $30 \mathrm{~d}$ with regular passaging and fixed in $70 \%$ ethanol. Cells were sequentially treated with RNase A $(500 \mu \mathrm{g} / \mathrm{ml})$ and PI $(100 \mu \mathrm{g} / \mathrm{ml}$ $\mathrm{PI})$. Cell cycle was detected with a FACScan flow cytometer (Becton Dickinson, USA) in triplicate.

\section{Long-term analysis on xenografted SW480 cells after lentivirus infection}

Four-week-old nude mice (athymic NCR-nu/nu) were purchased from Vital River (Beijing, China), and housed under germ-free conditions. SW480 cells infected with recombinant lentiviruses ( $\mathrm{MOI}$ $=20$ ) were harvested at 5 day after infection and suspended in PBS. Each mouse was subcutaneously injected with $2 \times 10^{7}$ cells ( $200 \mu \mathrm{l}$ in volume) at the right flank. The length and width of the tumors were measured with a caliper at 5-day intervals after the inoculation, and the tumor size was calculated using the formula: length $\times$ width $2 / 2$. Animals were sacrificed at 30 day after the infection, and the tumors were dissected and fixed in $4 \%$ polyformaldehyde for pathological observation on $\mathrm{H}+\mathrm{E}$ stained sections.

\section{Statistical analysis}

Data were evaluated by one-way ANOVA with SPSS software (Version 13.0). Value of $p<0.05$ was considered statistically significant. Results were expressed as the mean \pm SD. The mean values and standard deviations of at least 3 independent experiments are provided in all the figures.

\section{Results}

\section{Lentiviral vector production and transduction}

Three recombinant lentiviral vectors were constructed and a representative scheme of the lentivirus vector is presented in Figure $1 \mathrm{~A}$. The vector contained $6 \times$ His-tagged apoptin CDNA driven by pSur of different lengths, the SV40 polyA se- 
A

A $161 \mathrm{bp}$

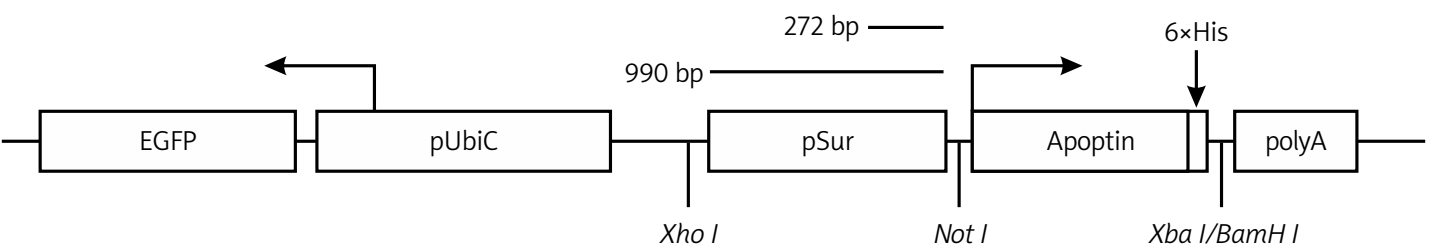

B

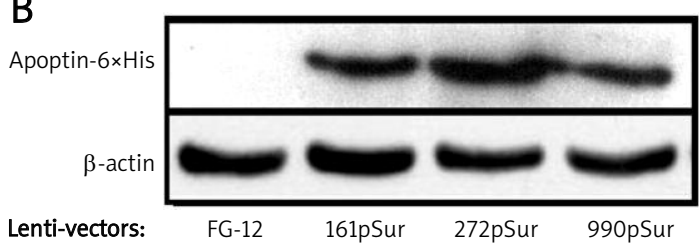

C

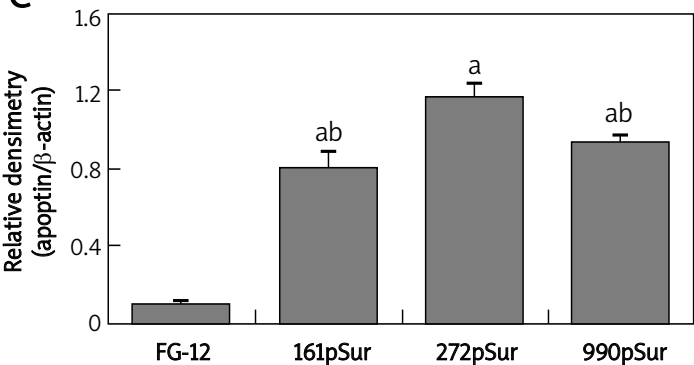

D

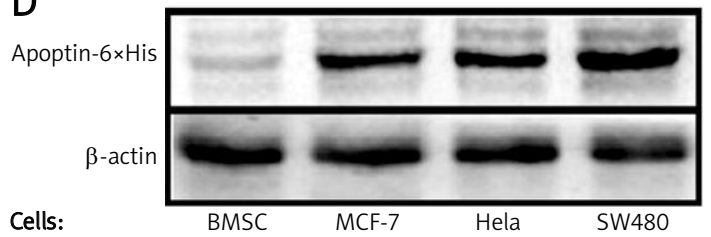

E

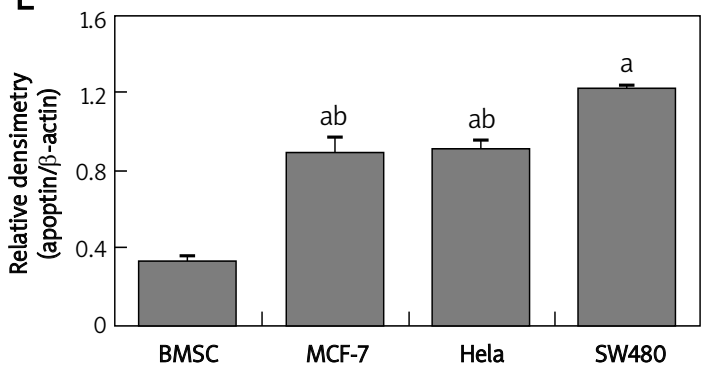

Figure 1. Activity comparison of pSur in different vectors and apoptin expression pattern in different cells. A-Scheme of the constructed lentivirus vector. B - Detection of apoptin-6xHis in 293FT cells transiently transfected with different lentiviral vectors. $C$ - Quantification of WB results. Since the transfection efficiency of different lentivirus vectors was similar, the expression level of apoptin- $6 \times$ His was adjusted by the expression level of $\beta$-actin: ${ }^{a} p<0.05$ vs. empty vector FG-12; ${ }^{b} p<0.05$ vs. FG-272pSur-ApoHis. D - Detection of apoptin-6xHis in different cells infected by lentivirus Lenti-272pSur-ApoHis. E - Quantification of WB results: ${ }^{a} p<0.05$ vs. BMSC; ${ }^{b} p<0.05$ vs. SW480 cells

quence, and the ubiquitin-C promoter-controlled GFP expression cassette for identification of the host cells. The introduction of the $6 \times$ His tag provided an epitope for protein detection.

Using 3 plasmid packaging system in 293FT cells, the recombinant lentivirus was generated and the self-inactivation of the viruses was ensured. After centrifuging concentration, the virus titer of the culture supernatant increased 50-100-fold, and the final virus titers were $1.45 \times 10^{8} \mathrm{TU} / \mathrm{m}$ for Lenti-FG12, $3 \times 10^{8} \mathrm{TU} / \mathrm{ml}$ for Lenti-161pSur-ApoHis, $2 \times 10^{8} \mathrm{TU} / \mathrm{ml}$ for Lenti-272pSur-ApoHis, and $2.5 \times 10^{8} \mathrm{TU} / \mathrm{ml}$ for Lenti-990pSur-ApoHis.

\section{Different activity of three pSur in lentiviral vectors}

Transient transfection of lentiviral vectors to 293FT cells was conducted to test the activity of the inserted expressing cassette. As the antibody against apoptin protein is not commercially available, we tagged the protein with $6 \times \mathrm{His}$ epitope to facilitate the detection. After the transfection, $85-90 \%$ of the cells showed GFP expression $24 \mathrm{~h}$ later and no obvious difference in the expression of $\beta$-gal was detected (data not shown), indicating a high and similar transfection efficacy. Western blot showed that 272 bp pSur possessed higher activity in the host cells since the expression level of apoptin-6xHis was almost 2-fold higher than the other two $(p<0.05)$. The transcriptional activity of pSur with $161 \mathrm{bp}$ and $990 \mathrm{bp}$ was comparable $(p>0.05)$ in 293 cells (Figures 1 B and 1 C).

\section{Differential expression pattern of 272pSur in lentivirus-infected cells}

To test the expression of pSur-controlled apoptin-6xHis, primary human BMSC, MCF-7, Hela and SW480 were infected by lentivirus Lenti-272pSurApoHis. The activity of the expression cassette was very weak in non-malignant cell BMSC, but was very high in tumor cells $(p<0.05)$. The expression of apoptin-6xHis protein in SW480 cells was even higher than that in MCF-7 and Hela cells $(p<0.05)$ (Figures $1 \mathrm{D}$ and $1 \mathrm{E}$ ).

\section{Apoptin-6xHis expression and its apoptosis-} inducing effect

The expression of apoptin- $6 \times$ His in SW480 cells was examined at 5 day and 30 day after infection. 
A

\begin{tabular}{|c|c|c|c|c|c|c|c|c|c|}
\hline \multirow{2}{*}{$\begin{array}{l}\text { Times after } \\
\text { infection }\end{array}$} & \multicolumn{4}{|c|}{ Day 5} & \multicolumn{4}{|c|}{ Day 30} & \\
\hline & 1 & 2 & 3 & 4 & 1 & 2 & 3 & 4 & \\
\hline $\begin{array}{r}6 \times \text { His-tagged } \\
\text { apoptin }\end{array}$ & & & & & & & & & $\leftarrow 14 \mathrm{kD}$ \\
\hline
\end{tabular}

B

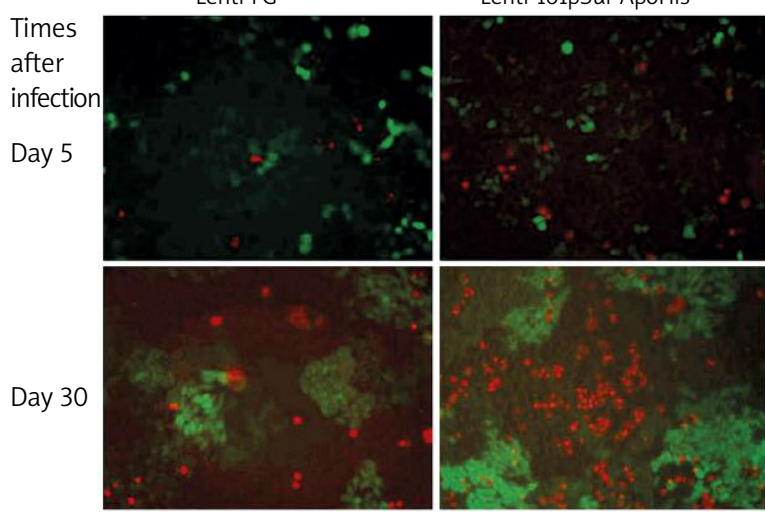

Lenti-272pSur-ApoHis

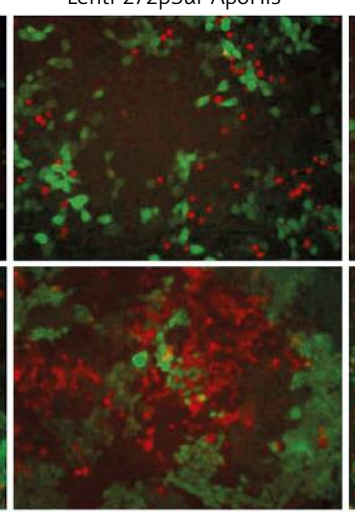

Lenti-990pSur-ApoHis

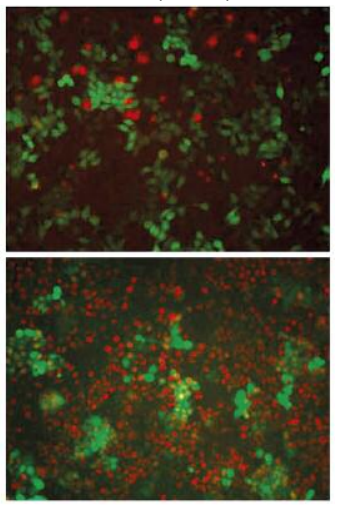

C

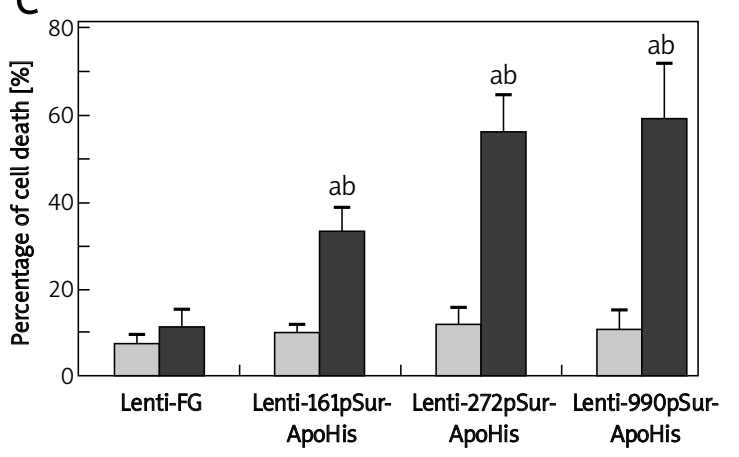

D

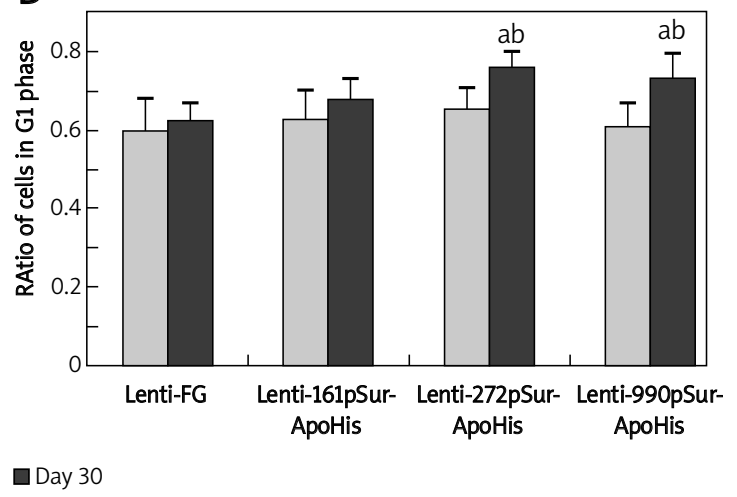

Figure 2. The apoptin expression driven by different fragmental pSur in SW480 and its suppressive effect on the cancer cells. A - The expression of apoptin-6xHis in SW480 cells detected by Western blot at 5 day and 30 day after the lentivirus infection: 1 - Lenti-FG; 2 - Lenti-990pSur-ApoHis; 3 - Lenti-272pSur-ApoHis; 4 - Lenti-161pSurApoHis. B - The apoptosis and necrosis revealed by dye binding under fluorescence microscope $($ bar $=100 \mu \mathrm{m})$ and C - the quantifying analysis of the dead cells. D - G1 phase ratio was examined by flow cytometry: ${ }^{a} p<0.05$ vs. day 5 group; ${ }^{b} p<0.05$ vs. control cells. All measurements were performed in triplicate

The protein blotting showed that all of the three pSur could drive apoptin-6xHis expression. The activity of 272pSur was almost 2 times higher than that of the other two promoters 5 day after the infection (Figure $2 \mathrm{~A}$ ), but at the same time, there were few apoptotic/necrotic cells presented (Figure 2 B, upper panel). At 30 day, the expression of apoptin-6xHis was not significantly different among the three types of lentivirus (Figure $2 \mathrm{~A}$ ). At this time, there appeared more apoptotic and necrotic cells, of which the dim GFP expression indicated the losing of cell vitality (Figure $2 \mathrm{~B}$, lower panel). Quantitative analysis confirmed higher cell death in Lenti-272pSur-ApoHis (56\%) and Lenti-990pSurApoHis (59\%) infected cells at day 30 than Lenti161 pur-ApoHis (33\%) induced cell death, which was significantly higher than in controls $(11 \%$, $p<0.05$ ) (Figure $2 \mathrm{C}$ ).

To further investigate the effect of recombinant lentivirus on cancer cell growth, the cell cycle was analyzed using flow cytometry. At 30 day after the infection, the G1 ratio of SW480 cells was significantly increased in Lenti-272pSur-ApoHis (76\%, $p$ $<0.05)$ and Lenti-990pSur-ApoHis $(73.5 \%, p<0.05)$ infected cells compared with control lentivirus (62.5\%). However, there was no significant difference among different lentivirus-infected cells at 5 day after infection (61-65.5\% among all treatments) (Figure 2 D). We hypothesize that timedependent apoptosis and cell cycle change induced by recombinant lentivirus are accumulative effects of the exogenous apoptin on the host cells. 


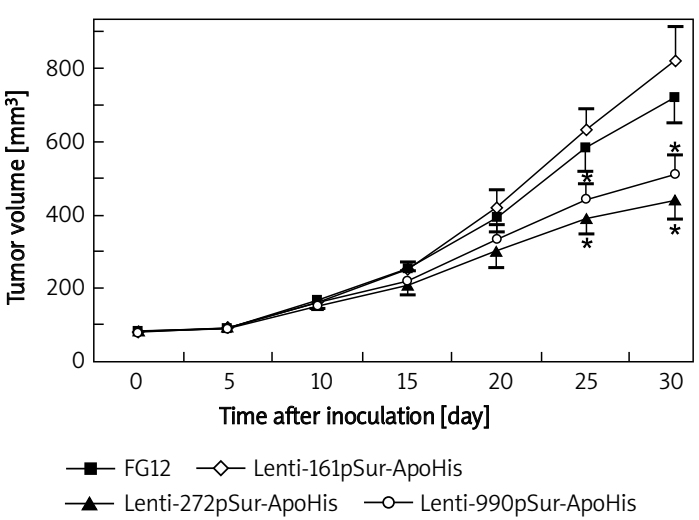

Figure 3. The infection of lentivirus expressing apoptin to SW480 cells suppressed the growth of xenografted tumors. Nude mice $(n=3-4$ mice per group) were inoculated with SW480 cancer cells infected with different viruses. Tumor size was measured and calculated every 5 day. Data are presented as mean \pm SD: a $p<0.05$ vs. control lentivirusinfected tumor cells

\section{Recombinant lentivirus expressing apoptin inhibited xenograft tumor growth}

Lentivirus-infected SW480 cells that carried different surviving promoter-driven apoptin expression cassettes were inoculated to nude mice and the growth of the xenografted tumor cells was measured every 5 day in vivo. The volume of the tumor increased with time, but there was no obvious difference among different cell populations within 20 day after the inoculation. At 25 day and 30 day, a significant slowdown of tumor growth appeared in Lenti-272pSur-ApoHis and Lenti990pSur-ApoHis infected cells when compared with control lentivirus and Lenti-161pSur-ApoHis infected cells $(p<0.05)$.

\section{Histology analysis of the xenograft tumors}

The xenograft tumors were collected 30 day after the inoculation and subjected to pathological observation. The control lentivirus-infected cells grew in a pattern of glandular structure-like or cell nests (Figure 4 A). Lentivirus Lenti-161pSur-ApoHis infected cells proliferated similarly except for the presence of vacant areas (white arrowheads) in the tumors and scattered apoptotic cells (black arrows) (Figure 4 B). In tumors derived from Lenti-161pSurApoHis (Figure 4 C) or Lenti-161pSur-ApoHis (Figure 4 D) infected cells, there were focal tissue necroses with different sizes (black arrowheads) and tissue structure was not recognizable. Tumor cells showed pyknotic nuclei and nuclear fragmentation.
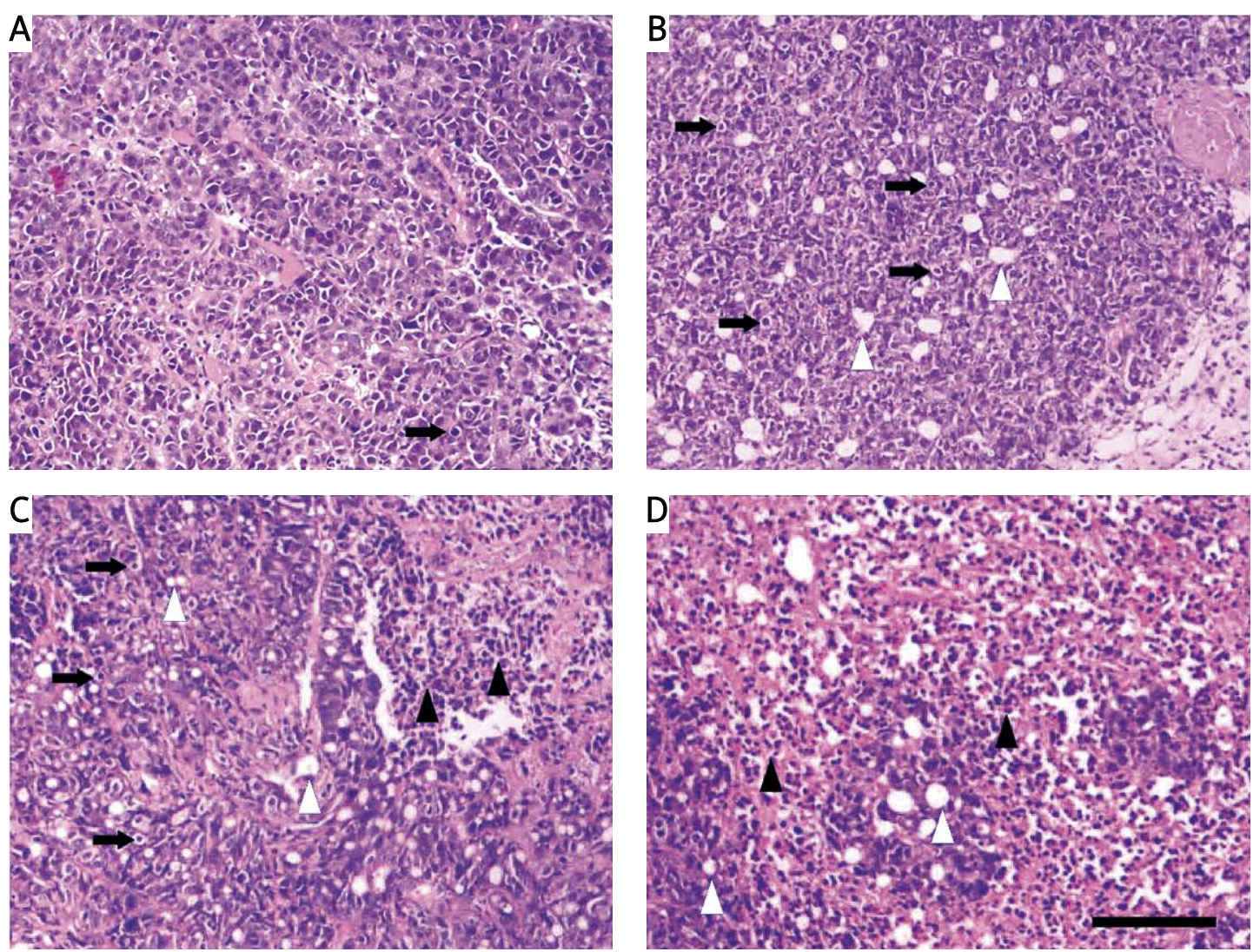

Figure 4. Pathological analysis of tumors derived from recombinant lentivirus-infected SW480 cells. A - Lenti-FG, B - Lenti-161pSur-ApoHis, C - Lenti-272pSur-ApoHis, and D - Lenti-990pSur-ApoHis infected cells were inoculated to nude mice

Black arrows - early apoptotic cells, black arrowheads - focal tissue necrosis with late apoptotic fragments, white arrowheads - vacant areas. Bar $=100 \mu \mathrm{m}$ 


\section{Discussion}

Experimental studies on tumor treatment using gene therapy have been conducted for years. Two critical issues have to be determined before the genetic manipulation on tumors: the gene(s) of interest and the promoter to drive the expression of the therapeutic gene(s). Apoptin has been previously applied in gene therapy under the control of highly active promoters, such as CMV promoter $[7,10,19]$, which may cause some unwanted results when applied in a long-lasting expression construct. Although it is reported that apoptin is a safe protein with few side effects on normal cells [3, 20, 21], tumor-specific promoter may improve the safety of gene therapy since it preferentially drives therapeutic gene expression in the scenario of targeted tumor cells in contrast to normal cells. In the present study, the promoter of survivin, a pan-tumor expressed gene $[7,8,10]$, was chosen to confine the expression of the exogenous apoptin in a proper population of cells. Because pSur is activated in most types of tumors with high specificity $[14,19$, $22,23]$, this strategy may have potential application in the treatment of a variety of tumors.

The basal transcription of survivin requires a proximal promoter region of $\sim 230 \mathrm{nt}$ from the initiating ATG, with several functional elements implicated in G1 transcriptional repression and several G2/M-regulated genes [24, 25]. This may be the reason why 161 bp pSur showed low activity both in vitro and in vivo among the three fragmental pSur. We demonstrated that the 272 bp and 990 bp pSur displayed comparable effects in terms of cell apoptosis/necrosis, G1 phase arrest, and tumor growth. Xu et al. obtained similar results in Hela cells [26]. It should be stated that the activity of these pSur fragments may vary with cell type. An individual pSur should be tested according to target cells, and our three pSur fragments provide options for proper pSur activity in certain cells.

We previously confirmed that the activity of pSur is much lower than CMV promoter even in malignant tumor cells [27], as in other reports [23, 28]. The natural pSur activity may result in a lowto-moderate level of apoptin to pursue cancer cell repression, and need a longer time to show its accumulative effect. This may reduce the possible side effects of exogenously expressed apoptin in normal cells. That the obvious death in lentivirusinfected cells occurred 30 day later may reflect the accumulative effect of the exogenous apoptin. In a xenograft tumorigenesis experiment in vivo, these two lentiviruses obviously repressed tumor growth, especially at the later stage (20-30 day after inoculation). Pathological analysis showed focal tumor tissue necrosis, and tumor cells showed pyknotic nuclei and nuclear fragmentation. In contrast, Lenti-161pSur-ApoHis infected cells only show scattered cell apoptosis and vacant areas in tumors.

The pSur provides the function to keep watch over quiescent cells infected with recombinant lentivirus and be active when cells divide. This may be practically useful in the elimination of malignantly transformed cells, since the pSur activity is ranked as cancer cells $>$ stem cells $>$ tissue cells [29]. We presume that activated pSur in malignant cells may induce a suicide event. This strategy of recombinant lentivirus construction also provides self-surveillance for transplanted cells in regenerative medicine, such as hematopoietic stem cells and mesenchymal stem cells [30]. Once malignant transformation occurs, this lentivirus system will initiate a self-clearance process, preventing any possible tumorigenesis of the transplanted cells.

In conclusion, the novel strategy of employing pSur and apoptin in lentivirus vector to ensure specific tumor cell repression is feasible. Our three recombinant lentiviruses with different tumor repression efficiency provide options for different manipulations in tumor cells. Equally importantly, this strategy can be applied to transplanted stem cells as a self-surveillance and self-clearance system.

\section{Acknowledgments}

Feng Ye and Bo Zhong contributed equally to this paper.

This work was supported by the National Natural Science Foundation of China (30828007), and the Natural Science Foundation Project of CQ CSTC (CSTC, 2007BA5011), China.

\section{References}

1. Danen-Van Oorschot AA, Fischer DF, Grimbergen JM, et al. Apoptin induces apoptosis in human transformed and malignant cells but not in normal cells. Proc Natl Acad Sci U S A 1997; 94: 5843-7.

2. Poon IK, Oro C, Dias MM, Zhang JP, Jans DA. A tumor cellspecific nuclear targeting signal within chicken anemia virus VP3/apoptin. J Virol 2005; 79: 1339-41.

3. Zhou S, Zhang M, Zhang J, Shen H, Tangsakar E, Wang J. Mechanisms of apoptin-induced cell death. Med Oncol 2012; 29: 2985-91.

4. Backendorf C, Visser AE, de Boer AG, et al. Apoptin: therapeutic potential of an early sensor of carcinogenic transformation. Annu Rev Pharmacol Toxicol 2008; 48: 143-69.

5. Noteborn MHM. Proteins selectively killing tumor cells. Eur J Pharmacol 2009; 625: 165-73.

6. Schoop RA, Kooistra K, Baatenburg De Jong RJ, Noteborn $\mathrm{MH}$. Bcl-xL inhibits p53- but not apoptin-induced apoptosis in head and neck squamous cell carcinoma cell line. Int J Cancer 2004; 109: 38-42.

7. Pietersen AM, van der Eb MM, Rademaker $\mathrm{HJ}$, et al. Specific tumor-cell killing with adenovirus vectors containing the apoptin gene. Gene Ther 1999; 6: 882-92. 
8. Pan Y, Fang L, Fan H, et al. Antitumor effects of a recombinant pseudotype baculovirus expressing apoptin in vitro and in vivo. Int J Cancer 2010; 126: 2741-51.

9. Mita AC, Mita MM, Nawrocki ST, Giles FJ. Survivin: key regulator of mitosis and apoptosis and novel target for cancer therapeutics. Clin Cancer Res 2008; 14: 5000-5.

10. Li X, Liu Y, Wen Z, et al. Potent anti-tumor effects of a dua specific oncolytic adenovirus expressing apoptin in vitro and in vivo. Mol Cancer 2010; 9: 10.

11. Van Houdt WJ, Haviv YS, Lu B, et al. The human survivin promoter: a novel transcriptional targeting strategy for treatment of glioma. J Neurosurg 2006; 104: 583-92.

12. Xiang J, Ouyang Y, Cui Y, et al. Silencing of Notch3 Using shRNA driven by survivin promoter inhibits growth and promotes apoptosis of human T-cell acute lymphoblastic leukemia cells. Clin Lymphoma Myeloma Leuk 2012; 12: 59-65.

13. Luo XR, Li JS, Niu Y, Miao L Targeted killing effects of double CD and TK suicide genes controlled by survivin promoter on gastric cancer cell. Mol Biol Rep 2011; 38: 1201-7.

14. Huang R, Zhao Z, Ma X, Li S, Gong R, Kuang A. Targeting of tumor radioiodine therapy by expression of the sodium iodide symporter under control of the survivin promoter. Cancer Gene Ther 2011; 18: 144-52.

15. Matrai J, Chuah MK, VandenDriessche T. Recent advances in lentiviral vector development and applications. Mol Ther 2010; 18: 477-90.

16. Liu GB, Cheng YX, Feng YK, et al. Adipose-derived stem cells promote peripheral nerve repair. Arch Med Sci 2011; 7: 592-6.

17. Hao L, Wang J, Zou Z, et al. Transplantation of BMSCs expressing hPDGF-A/hBD2 promotes wound healing in rats with combined radiation-wound injury. Gene Ther 2009; 16: 34-42.

18. Qin XF, An DS, Chen IS, Baltimore D. Inhibiting HIV-1 infection in human $T$ cells by lentiviral-mediated delivery of small interfering RNA against CCR5. Proc Natl Acad Sci USA 2003; 100: 183-8.

19. Lu B, Makhija SK, Nettelbeck DM, et al. Evaluation of tumor-specific promoter activities in melanoma. Gene Ther 2005; 12: 330-8.

20. Peng DJ, Sun J, Wang YZ, et al. Inhibition of hepatocarcinoma by systemic delivery of Apoptin gene via the hepatic asialoglycoprotein receptor. Cancer Gene Ther 2007; 14: 66-73.

21. Los M, Panigrahi S, Rashedi I, et al. Apoptin, a tumorselective killer. Biochim Biophys Acta 2009; 1793: 1335-42.

22. Garg H, Salcedo R, Trinchieri G, Blumenthal R. Improved nonviral cancer suicide gene therapy using survivin promoter-driven mutant Bax. Cancer Gene Ther 2010; 17: 155-63.

23. Zhu ZB, Makhija SK, Lu B, et al. Transcriptional targeting of tumors with a novel tumor-specific survivin promoter. Cancer Gene Ther 2004; 11: 256-62.

24. Li F, Altieri DC. Transcriptional analysis of human survivin gene expression. Biochem J 1999; 344: 305-11.

25. Li F, Ambrosini G, Chu EY, et al. Control of apoptosis and mitotic spindle checkpoint by survivin. Nature 1998; 396: 580-4.

26. Xu R, Zhang P, Huang J, Ge S, Lu J, Qian G. Sp1 and Sp3 regulate basal transcription of the survivin gene. Biochem Biophys Res Commun 2007; 356: 286-92.

27. Sun H, Wang M, Hao L, Wang J, Su Y, Zou Z. Human betadefensins improve wound healing through a mechanism affecting multi-aspects of healing process. In: Advances in wound care. Vol. 2. Sen CK (ed.). Mary Ann Liebert, Inc. Danbury, USA, 2011; 149-54.
28. Konopka K, Spain C, Yen A, Overlid N, Gebremedhin S, Duzgunes N. Correlation between the levels of survivin and survivin promoter-driven gene expression in cancer and non-cancer cells. Cell Mol Biol Lett 2009; 14: 70-89.

29. Fukuda S, Pelus LM. Survivin, a cancer target with an emerging role in normal adult tissues. Mol Cancer Ther 2006; 5: 1087-98.

30. Kashani IR, Golipoor Z, Akbari M, et al. Schwann-like cell differentiation from rat bone marrow stem cells. Arch Med Sci 2011; 7: 45-52. 